träger hält, durchgeführt. Benutzt wurde das Virus der Vergilbungskrankheit der Rübe (sugar beet yellows virus), das einige Tage in der Blattlaus vorhält und das am besten übertragen wird, wenn den für die Versuche benutzten Blattläusen mehrere Stdn. Gelegenheit zur Virusaufnahme und später zur Virusabgabe in die Testpflanzen gegeben wird. Es konnte bestätigt werden, daß Acyrthosiphon onobrychis (B. d. F.) Überträger der Vergilbungskrankheit der Rübe (2 von 10 Pflanzen infiziert) ist ${ }^{1}$. Auch Neomyzus circumflexus (B u c k t.) übertrug wieder die Vergilbungskrankheit (1 von 10 Pflanzen infiziert). Als neuer Überträger wurde Myzus portulacae ( $\mathrm{M} \mathrm{a} \mathrm{c} \mathrm{ch.)}=$ (Myzus ornatus L a ing) ermittelt. In drei Versuchsserien konnnte die Art insgesamt 16 von 30 Pflanzen infizieren, ein sehr beachtlicher Infektionserfolg, dessen Bedeutung allerdings für die deutschen Verhältnisse dadurch eingeschränkt wird, daß diese Blattlaus vorwiegend in Gewächshäusern und auf Zierpflanzen vorkommt. In wärmeren Gebieten kann die Art aber den Winter im Freiland überdauern. Da sie wohl annähernd 100 Wirtspflanzen befallen kann, beim Aufsuchen von Nährpflanzen also nicht sehr wählerisch ist, wird sie in wärmeren Gebieten des öfteren Gelegenheit haben, auf Pflanzen überzuwechseln, die für das Virus der Vergilbungskrankheit anfällig sind. Sie kann dann auf Unkräutern zum Entstehen von Virusherden beitragen, kann aber auch direkt das Virus auf Futter- oder Zuckerrüben übertragen. Begünstigt wird die Neuanstekkung von Pflanzen durch die relativ kurzen Saugzeiten es genügen wenige Min. - , die zur Übertragung ausreichend sind, wenn die Blattlaus infektiös ist. Semipersistente Viren dürften nicht nur äußerlich an den Stechborsten haften, sondern werden - ähnlich wie persistente Viren - den Körper des Überträgers passieren und mit dem Speichel in neue Pflanzen abgegeben.

1 W. Steudel u. A. Heiling, Mitt. biol. Zentralanst. Land- u. Forstwirtsch. Berlin-Dahlem 79, 1 [1954].

\title{
BESPRECHUNGEN
}

Ullmanns Encyklopädie der technischen Chemie. 3., völlig neu gestaltete Auflage. Herausgegeben von W. Foerst. 6. Band: Dimethyläther bis Extraktion. Verlag Urban u. Schwarzenberg, München 1955. XI, 827 S. mit 402 Abb.; Preis geb. DM 108.-.

Schon bei Besprechungen der früheren Bände * der Encyklopädie der Technischen Chemie wurde auf die sehr begrüßenswerte Tendenz des Herausgebers hingewiesen, wesensmäßig zusammengehörige Gebiete unter größeren Stichworten zu behandeln und diese übersichtlich aufzugliedern. Der Vorteil einer solchen Stoffeinteilung gegenüber dem Darbieten vieler über das Alphabet verteilter kurzer Stichworte wird im vorliegenden Band VI besonders deutlich; er enthält nur 24 Stichworte.

Für den technischen Gesichtspunkt des Gesamt-Werkes liegen in den großen Kapiteln „Eisen“ (ohne Stahl, $146 \mathrm{~S}$. und $187 \mathrm{Abb}$; mit eingehender Berücksichtigung der rein thermischen und thermodynamischen Grundlagen), „Email“ (22 S.), „Seltene Erden“ (32 S.) und vor allem „Erdgas und Erdöl“ (213 S. und $83 \mathrm{Abb}$.; ohne Petrochemikalien und die Verarbeitung von Primärprodukten zu Sekundärprodukten) naturgemäß die wichtigsten Beiträge vor. Aber auch im vorliegenden Bande kommt - wie in den früheren - der biologisch Interessierte voll auf seine Kosten. Besonders hingewiesen sei auf das Stichwort "Drogen“ (70 S.) ; sein Umfang erscheint für eine technische Encyklopädie ungewöhnlich groß. Aber solch ausführliche Darstellung ist eben bei allen Stichworten gewählt, die in die Nähe der Arzneimittelherstellung und der Pharmakotherapie rücken, und darum ist das Gesamtwerk so überaus nützlich, weit über den Kreis der Techniker hinaus!

Das Kapitel „Drogen“ enthält außer einer Beschreibung der Aufarbeitungsmethoden und einem Abschnitt

* 3. Band, Z. Naturforschg. 9 b, 623 [1954] ;

4. Band, Z. Naturforschg. 13 b, 475 [1958];

5. Band, Z. Naturforschg. 13 b, 631 [1958]. über Herstellung und Zubereitung der ArzneidrogenPräparate eine ausführliche Übersicht über die wichtigsten (etwa 400) pflanzlichen und tierischen Drogen, in der man viel Wissenswertes über Namen, Herkunft, Inhaltsstoffe und Verwendung erfährt. Auch das Stichwort "Düngemittel“ (88 S.) ist inhaltsreicher als man erwarten wird: einer geschichtlichen Einleitung folgen nicht nur technische Angaben über Anwendung und Wirkung der verschiedenen Düngeformen und über Art, Herstellung, Lagerung und Verpackung der verschiedenartigen Düngemittel, sondern man wird zusammenhängend über die Probleme der Pflanzenernährung, der Stoffbildung und des Wachstums unter Berücksichtigung von klimatischen und bodenwirksamen Wachstumsfaktoren und Hemmstoffen unterrichtet; ein Abschnitt über „Bodenkunde“ faßt viel Wissenswertes kurz zusammen. - Interesse wird auch das Kapitel über die Chemie der „Dithiocarbonsäuren und ihrer Derivate“ (9 S.) finden, die neben ihrer vielseitigen technischen Verwendung (Ultrabeschleuniger für die Bildung von natürlichem und künstlichem Kautschuk) Bedeutung als Fungicide und als analytische Hilfsmittel besitzen; substituierte Dithiocarbamate ersetzen $\mathrm{H}_{2} \mathrm{~S}$ in der Gruppenanalyse als Fällungsreagens und dienen zur colorimetrischen Cu-Bestimmung, besonders bei mikrochemischem Arbeiten mit biologischem Material. Steigende Bedeutung für Technik und Biochemie gewinnen die „Einschlußverbindungen“ (8 S.) ; sie sind Modelle für lockere Aneinanderlagerung verschiedener Molekeln durch gestaltliches Zueinanderpassen, und mit ihrer Hilfe lassen sich im Laboratorium und in der Technik Racemate trennen; möglicherweise spielt solch ein Vorgang eine Rolle bei der ersten Entstehung optisch aktiver Stoffe in der $\mathrm{Na}$ tur. - Dem Stichwort „Synthetische Edelsteine“ (33 S.) wird wohl mancher mit Interesse begegnen; an Stichworten allgemein informierenden Charakters enthält der Band „Elektrolyse“ (48 S.), „Emulsion“ (16 S.) und „Explosion“ (12 S.). 
$\mathrm{Zu}$ loben sind erneut die ausführlichen Literaturangaben und erstmalig ein Zwischenregister für die Bände 3-5, das deren Benutzung wesentlich erleichtert.

A. Butenandt, München.

Ullmanns Encyklopädie der technischen Chemie. 3., völlig neu gestaltete Auflage. Herausgegeben von W. FoERst. 7. Band: Färberei bis Gastrennung. Verlag Urban u. Schwarzenberg, München 1956. XI, 848 S. mit 341 Abb. und 4 farbigen Tafeln; Preis geb. DM 128.-.

Die Leser der Zeitschrift für Naturforschung sind durch voraufgegangene Besprechungen * der encyklopädischen Bände 3-6 des neuen ,Ullmann“ darüber unterrichtet, welch wertvolles Werk hier erscheint, das auch für den nur wissenschaftlich und biologisch interessierten Chemiker und für den Biologen als Nachschlagewerk vorzüglich geeignet ist. Das zeigt erneut der vorliegende 7 . Band.

Ref. hat besonders die Stichworte „Natürliche Farbstoffe" (40 S.), "Synthetische organische Farbstoffe“ (44 S.), „Farbstoffuntersuchung“ (46 S.), „Fermente“ (61 S.), „Fettalkohole“ (18 S.), „Fette und Fettsäuren“ (98 S.) und „Synthetische Fettsäuren“ (11 S.) durchgesehen. Die Farbstoffkapitel sind besonders gut gelungen. Die schöne Übersicht über die wichtigsten Naturfarbstoffe wird noch lange als verläßlicher Führer auf diesem Gebiet gelten können, wenn auch die neuere Entwicklung, die sich in einigen Sparten seit Niederschrift des Artikels vollzog, unsere Kenntnisse über neuere Naturfarbstoffe (z. B. Phenoxazone, Phenthiazone, Pteridine) wesentlich erweiterte. Einen vorzüglichen Überblick vermittelt das Stichwort „Synthetische organische Farbstoffe". Schon die ausführliche geschichtliche Tabelle ist eine Fundgrube, und der Abschnitt „Konstitution und Farbe“ zeugt von meisterhafter Beherrschung dieses interessanten Gebietes. Das Kapitel „Farbstoffuntersuchung“, das die neueren chromatographischen und physikalisch-optischen Verfahren neben den Methoden der Farbmessung berücksichtigt und mit anschaulichen Bunttafeln verdeutlicht, orientiert hervorragend. Dasselbe kann man über die ausführlichen $\mathrm{Ab}$ schnitte sagen, die den Fettstoffen gewidmet sind; sie sind vom wissenschaftlichen wie vom technischen $\mathrm{Ge}$ sichtspunkt aus gleich ausgewogen abgefaßt.

Leider erweist sich der Text zum Stichwort „Fermente“ demgegenüber als uneinheitlich; hier fühlte sich der Referent bei seinen „Ullmann“-Studien erstmalig enttäuscht. Das Gebiet ist von allzu vielen Autoren bearbeitet worden; damit gewann man zwar gut gelungene Einzelabschnitte, vor allem zur Technologie der Fermente, aber der UUberblick über das Gesamtgebiet ist nicht geglückt, die Auswahl der in den allgemeinen Abschnitten behandelten Fermente und ihrer Analytik erscheint nicht begründet, und die Betrachtungsart ist an manchen Stellen veraltet. Eine Encyklopädie ist auch

* 3. Band, Z. Naturforschg. 9 b, 623 [1954];

4. Band, Z. Naturforschg. 13 b, 475 [1958];

5. Band, Z. Naturforschg. 13 b, 631 [1958];

6. Band, Z. Naturforschg. 14 b, 415 [1959]. kaum der Ort, neue Nomenklaturvorschläge zu machen, die noch der eingehenden Diskussion bedürfen.

Unter anderen Stichworten findet man wieder viel Wissenswertes, es sei besonders auf die Abschnitte „Färberei“, „Fasern und Borsten“ (mit einem übersichtlichen Abschnitt über Faserstrukturen!) und „Futtermittel" hingewiesen. Literaturübersichten und Ausstattung sind von bewährter Güte.

A. Butenandt, München.

A modern Approach to Organic Chemistry. Von J. PAcker und J. Vaughan. Verlag Oxford University Press, London 1958. X, 973 S.; Preis geb. 84s.net.

$\mathrm{Zu}$ einer Zeit, in der auch bei uns das Gespräch über Ausbildungsfragen in Chemie besonders lebhaft geführt wird, ist das Buch von J. Packer und J. Vaughan „Eine moderne Einführung in die organische Chemie" besonders aktuell und anregend. Im Gegensatz zu anderen angelsächsischen Autoren, welche die organische Chemie abstrakt und durch die Brille einer noch in der Entwicklung stehenden Theorie betrachtet haben, versuchen die beiden Autoren einen Ausgleich zu finden zwischen der Vielfalt der stofflichen Erscheinungen und der Theorie.

Es soll vorweggenommen werden, daß dies in hohem Maße gelungen ist, allerdings unter radikaler Beschränkung des abgehandelten Stoffes auf Fundamentalzusammenhänge. Zum Beispiel sind die Monosaccharide und Aminosäuren sehr ausführlich und sachkundig dargestellt, während über Polysaccharide oder Polypeptide so gut wie nichts zu finden ist. Dies gilt für alle Naturstoffe, die bestenfalls in einer Fußnote erwähnt sind. Diese Stoffbeschränkung, so unzulässig sie vielleicht vom konservativen Fachkollegen empfunden wird, gibt dem Buch durch die Einheitlichkeit der Thematik seinen besonderen didaktischen Wert und seine innere Geschlossenheit.

Nach einer modernen und gut verständlichen Einführung in das Wesen der chemischen Bindung auf knapp 20 Seiten beschreiben die Autoren sehr systematisch die einzelnen Stoffklassen und erläutern in didaktisch ausgezeichneter Weise am Objekt die Theorie. Durch diese lebendige Wechselbeziehung zwischen Experimentalbefund und Theorie wird dem Leser als einer Art höherer Einheit das Gefühl vermittelt, daß er nicht mehr gedächtnismäßig lernt, sondern daß er „versteht". Die theoretischen Grundlagen sind damit nicht nur Mittel zum Verständnis von Einzelfakten geworden, sondern zu einem dynamischen Prinzip der Stoffordnung.

Recht angenehm wirkt der klare Stil, die einfachen Sätze, die wertvollen Hinweise auf Querbeziehungen und die kurze und prägnante Erläuterung der Nomenklatur. An der Auswahl der Beispiele, an der die Theorie erarbeitet wird, erkennt man die langjährigen, erfahrenen Pädagogen, die immer bemüht sind, die Grenzen des Gesicherten sichtbar werden zu lassen und ausdrücklich darauf hinweisen, wenn sie übersimplifizieren. Überall sind interessante präparative Hinweise eingestreut, und manchmal neueste Methoden genannt, die auch dem schon erfahrenen Chemiker die Lektüre interessant machen. Der deutsche Leser wird ferner mit 
Befriedigung feststellen, daß nicht nur angelsächsische Autoren genannt sind. Der Referent hat nur erfreulich wenige Druckfehler gefunden und kann den Druck und die Gesamtanordnung des gebotenen Stoffes nur lobend hervorheben.

In unserem deutschen Schrifttum fehlt ein Buch, welches in so ausgewogener Form einen Querschnitt durch Stoff und Theorie vermittelt. Der interessierte Chemiestudent wird in der Zeit seines organisch-chemischen Praktikums, aber auch später, dieses Buch mit ganz großem Gewinn zur Hand nehmen und dadurch eine Fülle beglückender Einsichten gewinnen können.

L. Horner, Mainz.

Der lichtelektrische Effekt und seine Anwendungen. 2. Auflage. Herausgegeben von H. Simon und R. Suhrmann. Springer-Verlag, Berlin 1958. XII, 747 S. mit 599 Abb.; Preis geb. DM 97.50.

Der jetzt nach einem Zeitraum von 25 Jahren erschienenen zweiten Auflage muß schon deswegen eine besondere Bedeutung zuerkannt werden, weil der lichtelektrische Effekt in den letzten Jahrzehnten ein entscheidender Bestandteil der wissenschaftlichen, aber auch der technischen Weiterentwicklung geworden ist. Die Namen der Autoren bieten die Garantie für ein hohes wissenschaftliches Niveau. Trotzdem ist, was den Wert des Werkes im Hinblick auf eine weite Verbreitung noch besonders erhöht, die Darstellung so klar und verständlich, daß es für die vielen auf diesem Gebiet Arbeitenden zu einem unentbehrlichen Rüstzeug werden wird.

Die Gliederung ist glücklich gewählt. Nach einer kurzen allgemeinen Einleitung beschreibt R. Suhrmann zunächst den äußeren lichtelektrischen Effekt und anschließend K. W. Böer die inneren lichtelektrischen Effekte. Es folgen zwei Abschnitte von H. Simon über die Herstellung von Photozellen mit äußerem Effekt, von Photowiderständen und Photoelementen (Halbleiterzellen). F. Eckart beschreibt sodann die SekundärelektronenVerstärkung und W. Leo und R. Suhrmann Methoden und Apparate bei lichtelektrischen Messungen. Die verschiedenen Anwendungen der Photozelle runden die Darstellung ab (W. Leo und R. Suhrmann, Photometrie; F. EcKart, Elektronenoptische Bildwandler, Röntgenbildverstärker, Fernsehtechnik und SekundärelektronenVervielfacher). Den Abschluß bilden Ausführungen von W. Leo und R. Simon über besondere Anwendungsgebiete der Photozelle, wie Tonfilm, Signalgebung, Zeitmessung, Dosimetrie und Umwandlung von Sonnenstrahlen in elektrische Energie. Das Buch kann als Standardwerk von größter Aktualität bezeichnet werden.

F. Töрт, Berlin.

Neue Ergebnisse der Nervenphysiologie. 6 Vorträge. Von Alexander v. Muralt. Springer-Verlag, Berlin-Wilmersdorf 1958. XI, 269 S. mit 158 Abb.; Preis geb. DM 49.80.

Die Fortschritte auf dem Gebiet der Nervenphysiologie waren in den letzten zwei Jahrzehnten besonders groß. In deutscher Sprache fand der Interessierte bislang keine umfangreichere moderne Zusammenfassung, er blieb auf vereinzelte Übersichtsreferate angewiesen. Um so erfreulicher ist das Erscheinen dieses ausgezeichneten Buches von einem Autor, der selbst großen Anteil an der stürmischen Entwicklung der Nervenphysiologie hat. Den Leser erwartet kein Lehrbuch oder eine Fachmonographie, sondern die lebendige Diskussion des letzten Standes der Forschung. Das Buch entstand aus der Überarbeitung und Zusammenfassung einer Zahl von Vorträgen, die Verf. in den Jahren 1955 - 57 gehalten hat. Man spürt so das Bemühen des Autors, den Hörer und Leser für dieses interessante Gebiet der Physiologie $\mathrm{zu}$ begeistern; die Darstellung ist aufgelockert und flüssig geblieben. Nachahmenswert ist die faire Diskussion aller, auch der nicht allgemein anerkannten Theorien und Hypothesen, sofern sie überhaupt ernst zu nehmen sind.

Im einzelnen werden ausführlich behandelt: 1. Ein geschichtlicher Überblick. 2. Die Stoffwechselvorgänge bei der Nervenerregung. 3. Die Ionentheorie der Erregung. 4. Die saltatorische Leitung. Im 5. und 6. Abschnitt folgen zwei meist weniger beachtete Gebiete, nämlich Untersuchungen über die Rolle des Aneurins bei der Nervenerregung und schließlich die Vorgänge bei der Degeneration und Regeneration des peripheren Nerven. Den Schluß bildet ein gemeinsam mit S. WeIDMANN bearbeiteter Abschnitt, in dem stichwortartig die Methoden der Nervenphysiologie behandelt sind. Die Darstellung der Ionentheorie der Erregung und der saltatorischen Leitung ist in sich geschlossen und vermittelt joweils einen ausgezeichneten Überblick über die gesamte Theorie. Das zweite, fünfte und sechste Kapitel führen naturgemäß nur in die Problematik der zur Zeit sehr im Fluß befindlichen Dinge ein. Die Abschnitte über Stoffwechselvorgänge und über die Rolle des Aneurins setzen beim Leser gewisse Kenntnisse der Physiologischen Chemie voraus, während bei den anderen $\mathrm{Ka}$ piteln auch der Außenstehende relativ leicht folgen können wird. Etwas bedauert hat der Ref., daß der ausgewählte Stoff so ausschließlich auf die Physiologie der Nervenfaser beschränkt wurde; ein Kapitel über die Physiologie der Ganglienzelle, der Synapsen und Sinneszellen wäre eine wertvolle Ergänzung der sonst reichhaltigen Darstellung gewesen.

$\mathrm{Zu}$ erwähnen ist die Ausstattung des Buches: Zahlreiche Tabellen, Zahlenangaben, der methodische Abschnitt und die Vielzahl der didaktisch hervorragenden Abbildungen machen das Werk zu einer Fundgrube an Material für Vorlesungen und Kurse.

\section{Burkhardt, München.}

Recent Progress in Hormone Research. Vol XIV. Proceedings of the Laurentian Hormone Conference 1957. Herausgegeben von Gregory Pincus. Verlag Academic Press Inc., New York 1958; in Deutschland durch Minerva, G.m.b.H., Frankfurt/Main. VI, 582 S. mit mehreren Abb.; Preis geb.U S-\$ 13.50.

Eine besondere Einführung erübrigt sich bei dieser bekannten Reihe, die mit den kritischen, oft persönlich gefärbten Vorträgen der Laurentian Hormone Conference eine erfreuliche Ergänzung zu den nur referierenden Fortschrittsberichten geben. Der vorliegende Band 
behandelt unter I. „Struktur und Funktion der Hormone“ (drei Vorträge), vor allem neue, synthetisch oder partialsynthetisch gewonnene Verbindungen; unter II. "Hormone bei Wachstum und Entwicklung" die Wirkung auf Gewichtszunahme und Nahrungsausnutzung, die auch praktisch in der Viehzucht Anwendung findet. Der III. Abschnitt enthält zwei Vorträge über Mißbildungen der Geschlechtsorgane, ihre Beziehung zum "chromosomalen Geschlecht" und ihre klinische Behandlung, wobei sich ergibt, daß bei Pseudohermaphroditismus das chromosomale Geschlecht nicht immer wegleitend für die Behandlung ist. Der IV. Abschnitt „Hormone und Stoffwechsel" bringt u. a. zwei Referate über das Bindegewebe, das beim Altern charakteristische Veränderungen erleidet; im Verhältnis Hexosamin : Collagen konnte ein $\mathrm{Maß}$ für das „biochemische Alter“ gefunden und mit der Ausscheidung von Hormonmetaboliten im Harn (als Index des Hormonspiegels) korreliert werden. Der V. Abschnitt (zwei Vorträge) ist den neurohumoralen Überträgersubstanzen Adrenalin und Noradrenalin gewidmet.

Auffallend an diesem Band ist der große Anteil medizinisch-praktisch orientierter Beiträge; die Grundlagenforschung kommt diesmal etwas zu kurz weg, obwohl gerade diese Beiträge, z. B. zum Wirkungsmechanismus der Östrogene (G. Mueller et al.), über Lactation (Lyons, Li und Johnson) und über die Verteilung der Neurohormone (U. S. v. Euler) von besonders hohem Standard sind. Die ausführlich wiedergegebenen Diskussionen erhöhen den Wert des Buches beträchtlich. - Druck und Ausstattung, auch die Wiedergabe der Photographien, sind ausgezeichnet.

P. Karlson, München.

Evolution by Natural Selection. Von Ch. Darwin und A. R.

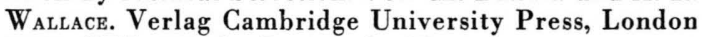
1958. VIII, 288 S.; Preis geb. 25 s.net.

Im Jahre 1842, also 6 Jahre nach der Rückkehr von seiner knapp 5-jährigen Weltreise, schrieb Charles DArwin als 33-jähriger zum ersten Mal seine Selektionstheorie in geschlossener Form nieder. 1844 verfaßte er einen neuen, erweiterten Aufsatz über das gleiche Thema und vertraute das Manuskript seiner Frau mit der Bitte an, es im Falle seines Todes zu veröffentlichen. Erst 14 Jahre später, im Jahre 1858, legte Darwin, gleichzeitig mit Alfred Russel Wallace, seine Theorie der wissenschaftlichen Öffentlichkeit vor. Ein Jahr später erschien dann sein großes Werk "The Origin of Species by means of Natural Selection". - Das Buch enthält die Dokumente von 1842, 1844 und 1858. Ihnen gehen ein ausgezeichneter, knapper Abriß (22 S.) der Geschichte der Evolutionslehre von Darwins Weltreise bis zur Gegenwart von Sir Gavin de BeER sowie ein biographischer Aufsatz von Charles Darwins Sohn, Sir Francis Darwin, voran. B. Hassenstein, Tübingen.

The Experimental Control of Plant Growth. Von Frits W. Went. Verlag Ronald Press Company, New York 1957. XVII, 343 S. mit mehreren Abb.; Preis geb. US-\$ 8.50 .

Die „Experimentelle Kontrolle des Wachstums der Pflanzen" ist keine systematische Darstellung ökologischer bzw. physiologischer Probleme, sondern ein Bericht über die Earhart-Laboratorien und die seit deren Fertigstellung durchgeführten Untersuchungen.

Das Buch, dessen größten Teil Went selbst geschrieben und durch fünf kleinere Kapitel anderer Autoren ergänzt hat, betrifft in seinem ersten Teil den Aufbau, die Einrichtung und den Betrieb des „Phytotrons“. Der zweite Teil - vom Autor selbst auch als die Ökologie einzelner Pflanzen bezeichnet - enthält eine Zusammenstellung der Ergebnisse zahlreicher Untersuchungen über die Reaktion einer großen Anzahl von Kultur- und einer kleineren Zahl von Wildpflanzen (hauptsächlich Wüsten- und Chaparralpflanzen) auf Außenfaktoren. Dabei wird die Wirkung von Licht und Temperatur besonders berücksichtigt.

In der abschließenden, sehr umfangreichen „Allgemeinen Diskussion“ (general discussion) entwickelt der Autor seine Konzeption über das Zusammenwirken von inneren und äußeren Faktoren beim Wachstum und bei der Entwicklung im weitesten Sinne. Den Schwerpunkt dieses Teiles bilden die Kapitel über Wachstum. Klimatologie und Ökologie.

Charakteristisch für dieses Buch, das zahlreiche graphische Darstellungen enthält, ist der subjektive Zug, der sich vor allem in der Interpretation der Versuchsergebnisse, aber auch darin ausprägt, da $\beta$ die zu den verschiedenen Problemen vorliegende Literatur nicht berücksichtigt wird. Das hat seine positiven Seiten darin, daß in vielen Fällen wertvolle neue Gesichtspunkte, bei manchen Fragen allerdings auch zu subjektive Ideen vertreten werden. Diese Kritik, die neben der Ansicht Wents über die Wirkung von Regenfällen auf die Keimung auch Fragen des Zuckertransports und der Rolle des Auxins beim Wachstum betrifft, soll und kann den Wert dieses vielseitigen und anregenden Buches nicht beeinträchtigen, das für Ökologen, Pflanzengeographen und Physiologen äußerst interessant ist.

J. Reinert, Tübingen. 